\title{
On the maximum size of a $(k, l)$-sum-free subset of an abelian group
}

\author{
Béla Bajnok \\ Department of Mathematics, Gettysburg College \\ Gettysburg, PA 17325-1486 USA \\ E-mail: bbajnok@gettysburg.edu
}

September 25, 2007

\begin{abstract}
A subset $A$ of a given finite abelian group $G$ is called $(k, l)$-sum-free if the sum of $k$ (not necessarily distinct) elements of $A$ does not equal the sum of $l$ (not necessarily distinct) elements of $A$. We are interested in finding the maximum size $\lambda_{k, l}(G)$ of a $(k, l)$-sum-free subset in $G$.

A $(2,1)$-sum-free set is simply called a sum-free set. The maximum size of a sum-free set in the cyclic group $\mathbb{Z}_{n}$ was found almost forty years ago by Diamanda and Yap; the general case for arbitrary finite abelian groups was recently settled by Green and Ruzsa. Here we find the value of $\lambda_{3,1}\left(\mathbb{Z}_{n}\right)$. More generally, a recent paper of Hamidoune and Plagne examines $(k, l)$-sum-free sets in $G$ when $k-l$ and the order of $G$ are relatively prime; we extend their results to see what happens without this assumption.
\end{abstract}

2000 Mathematics Subject Classification:

Primary: 11P70;

Secondary: 05D99, 11B25, 11B75, $20 \mathrm{~K} 01$.

Key words and phrases:

Sum-free sets, $(k, l)$-sum-free sets, Kneser's Theorem, arithmetic progressions.

\section{Introduction}

Throughout this paper, we let $G$ be a finite abelian group of order $n>1$, written in additive notation; $v$ will denote the exponent (i.e. largest order of any element) of $G$.

For subsets $A$ and $B$ of $G$, we use the standard notations $A+B$ and $A-B$ to denote the set of all two-term sums and differences, respectively, with one term chosen from $A$ and one from $B$. If, 
say, $A$ consists of a single element $a$, then we simply write $a+B$ and $a-B$ instead of $A+B$ and $A-B$. For a positive integer $h$ and a subset $A$ of $G$, the set of all $h$-term sums with (not necessarily distinct) elements from $A$ will be denoted by $h A$.

Let $k$ and $l$ be distinct positive integers. A subset $A$ of $G$ is called a $(k, l)$-sum-free set in $G$ if

$$
k A \cap l A=\emptyset ;
$$

or, equivalently, if

$$
0 \notin k A-l A .
$$

Clearly, we may assume that $k>l$. We are interested in determining the maximum possible size $\lambda_{k, l}(G)$ of a $(k, l)$-sum-free set in $G$.

A $(2,1)$-sum-free set is simply called a sum-free set. The value of $\lambda_{2,1}\left(\mathbb{Z}_{n}\right)$ was determined by Diamanda and Yap [13 in 1969. It can be proved (see also [31) that

$$
\max _{d \mid v}\left\{\left\lfloor\frac{d+1}{3}\right\rfloor \cdot \frac{n}{d}\right\} \leq \lambda_{2,1}(G) \leq \max _{d \mid n}\left\{\left\lfloor\frac{d+1}{3}\right\rfloor \cdot \frac{n}{d}\right\},
$$

which for cyclic groups immediately implies the following.

Theorem 1 (Diamanda and Yap [13]) The maximum size $\lambda_{2,1}\left(\mathbb{Z}_{n}\right)$ of a sum-free set in the cyclic group of order $n$ is given by

$$
\lambda_{2,1}\left(\mathbb{Z}_{n}\right)=\max _{d \mid n}\left\{\left\lfloor\frac{d+1}{3}\right\rfloor \cdot \frac{n}{d}\right\}= \begin{cases}\frac{p+1}{p} \cdot \frac{n}{3} \quad \begin{array}{l}
\text { if } n \text { is divisible by a prime } p \equiv 2(\bmod 3) \\
\text { and } p \text { is the smallest such prime; }
\end{array} \\
\left\lfloor\frac{n}{3}\right\rfloor \quad \text { otherwise. }\end{cases}
$$

The problem of finding $\lambda_{2,1}(G)$ for arbitrary $G$ stood open for over 35 years. In a recent breakthrough paper, Green and Ruzsa [15] proved that, as it has been conjectured, the value of $\lambda_{2,1}(G)$ agrees with the lower bound in (11):

Theorem 2 (Green and Ruzsa [15]) The maximum size $\lambda_{2,1}(G)$ of a sum-free set in $G$ is

$$
\lambda_{2,1}(G)=\lambda_{2,1}\left(\mathbb{Z}_{v}\right) \cdot \frac{n}{v}=\max _{d \mid v}\left\{\left\lfloor\frac{d+1}{3}\right\rfloor \cdot \frac{n}{d}\right\} .
$$

As a consequence, we see that

$$
\frac{2}{7} n \leq \lambda_{2,1}(G) \leq \frac{1}{2} n
$$

for every $G$, with equality holding in the lower bound when $v=7$ and in the upper bound when $v$ (iff $n$ ) is even.

Now let us consider other values of $k$ and $l$. In Section 2 of this paper we generalize (11), and prove the following. 
Theorem 3 The maximum size $\lambda_{k, l}(G)$ of a $(k, l)$-sum-free set in $G$ satisfies

$$
\max _{d \mid v}\left\{\left(\left\lfloor\frac{d-1-\delta(d)}{k+l}\right\rfloor+1\right) \cdot \frac{n}{d}\right\} \leq \lambda_{k, l}(G) \leq \max _{d \mid n}\left\{\left(\left\lfloor\frac{d-2}{k+l}\right\rfloor+1\right) \cdot \frac{n}{d}\right\}
$$

where $\delta(d)=\operatorname{gcd}(d, k-l)$.

Note that for $(k, l)=(2,1)$ Theorem 3 yields (1). Note also that, if $k-l$ is not divisible by $v$, then $\delta(v)=\operatorname{gcd}(v, k-l) \leq v / 2$; in particular,

$$
\lambda_{k, l}(G) \geq \frac{n}{2(k+l)}>0 .
$$

If, on the other hand, $k-l$ is divisible by $v$, then clearly $\lambda_{k, l}(G)=0$, since for any $a \in G$ we have $k a=l a$.

Let us now consider cyclic groups. When $G \cong \mathbb{Z}_{n}$ and $n$ and $k-l$ are relatively prime, then Theorem 3 gives

$$
\lambda_{k, l}\left(\mathbb{Z}_{n}\right)=\max _{d \mid n}\left\{\left(\left\lfloor\frac{d-2}{k+l}\right\rfloor+1\right) \cdot \frac{n}{d}\right\} .
$$

This result was already established by Hamidoune and Plagne in 17]. Their method was based on a generalization of Vosper's Theorem [30] on critical pairs where arithmetic progressions, that is, sets of the form

$$
A=\{a, a+d, \ldots, a+c \cdot d\}
$$

play a crucial role. In particular, Hamidoune and Plagne proved that, if $G \cong \mathbb{Z}_{n}$ and $n$ and $k-l$ are relatively prime, then

$$
\lambda_{k, l}\left(\mathbb{Z}_{n}\right)=\max _{d \mid n}\left\{\alpha_{k, l}\left(\mathbb{Z}_{d}\right) \cdot \frac{n}{d}\right\},
$$

where $\alpha_{k, l}\left(\mathbb{Z}_{n}\right)$ is the maximum size of a $(k, l)$-sum-free arithmetic progression in $\mathbb{Z}_{n}$. Hamidoune and Plagne deal only with the case when $n$ and $k-l$ are relatively prime; as they point out, "in the absence of this assumption, degenerate behaviors may appear", and we concur with this assessment. Nevertheless, we attempt to treat the general case; in Section 3 of this paper we prove that (3) remains valid even without the assumption that $n$ and $k-l$ are relatively prime:

Theorem 4 For arbitrary positive integers $k, l$, and $n$ we have

$$
\lambda_{k, l}\left(\mathbb{Z}_{n}\right)=\max _{d \mid n}\left\{\alpha_{k, l}\left(\mathbb{Z}_{d}\right) \cdot \frac{n}{d}\right\}
$$

Let us now move on to general abelian groups. Hamidoune and Plagne conjecture in [17] that

$$
\lambda_{k, l}(G)=\lambda_{k, l}\left(\mathbb{Z}_{v}\right) \cdot \frac{n}{v}
$$

holds when $n$ and $k-l$ are relatively prime. They prove this assertion with the additional assumption that at least one prime divisor of $v$ is not congruent to $1(\bmod k+l)$. We generalize this result for the case when $n$ and $k-l$ are not necessarily relatively prime: 
Theorem 5 As before, for a positive integer $d$, we set $\delta(d)=\operatorname{gcd}(d, k-l)$. If $v$ possesses at least one divisor $d$ which is not congruent to any integer between 1 and $\delta(d)$ (inclusive) $(\bmod k+l)$, then

$$
\lambda_{k, l}(G)=\lambda_{k, l}\left(\mathbb{Z}_{v}\right) \cdot \frac{n}{v} .
$$

We closely follow some of the fundamental work of Hamidoune and Plagne in 17; ; in fact, Section 3 of this paper can be considered an extention of [17] for the case when $n$ and $k-l$ are not assumed to be relatively prime.

In Section 4 we employ Theorem 4 to establish the value of $\lambda_{3,1}\left(\mathbb{Z}_{n}\right)$ explicitly. As an analogue to Theorem 1 we prove the following.

Theorem 6 The maximum size $\lambda_{3,1}\left(\mathbb{Z}_{n}\right)$ of a $(3,1)$-sum-free set in the cyclic group of order $n$ is given by

$$
\lambda_{3,1}\left(\mathbb{Z}_{n}\right)=\max _{d \mid n}^{d \neq 2(\bmod 4)}\left\{\left\{\left\lfloor\frac{d+2}{4}\right\rfloor \cdot \frac{n}{d}\right\}=\left\{\begin{array}{cc}
\frac{p+1}{p} \cdot \frac{n}{4} & \text { if } n \text { is divisible by a prime } p \equiv 3(\bmod 4) \\
& \text { and } p \text { is the smallest such prime; } \\
\left\lfloor\frac{n}{4}\right\rfloor \quad & \text { otherwise. }
\end{array}\right.\right.
$$

As a consequence, we see that

$$
\frac{1}{5} n \leq \lambda_{3,1}\left(\mathbb{Z}_{n}\right) \leq \frac{1}{3} n,
$$

with equality holding in the lower bound when $n \in\{5,10\}$ and in the upper bound when $n$ is divisible by 3 .

In our final section, Section 5, we provide some further comments and discuss several open questions about $(k, l)$-sum-free sets.

\section{Bounds for the size of maximum $(k, l)$-sum-free sets}

In this section we prove Theorem 3 ,

We will use the following easy lemma.

Lemma 7 Suppose that $A$ is a maximal $(k, l)$-sum-free set in $G$. Let $K$ denote the stabilizer subgroup of $k A$. Then

(i) $k(A+K)=k A$;

(ii) $A+K$ is a $(k, l)$-sum-free set in $G$;

(iii) $A+K=A$;

(iv) $A$ is the union of cosets of $K$. 
Proof. (i) The inclusion $k A \subseteq k(A+K)$ is obvious. Suppose that $a_{1}, \ldots, a_{k} \in A$ and $h_{1}, \ldots, h_{k} \in$ $K$. Then

$$
\left(a_{1}+\cdots+a_{k}\right)+\left(h_{1}+\cdots+h_{k}\right) \in k A,
$$

so $k(A+K) \subseteq k A$.

(ii) Suppose, indirectly, that

$$
k(A+K) \cap l(A+K) \neq \emptyset ;
$$

by (i) this implies

$$
k A \cap l(A+K) \neq \emptyset .
$$

Then we can find elements $a_{1}, \ldots, a_{k} \in A, a_{1}^{\prime}, \ldots, a_{l}^{\prime} \in A$, and $h_{1}, \ldots, h_{l} \in K$ for which

$$
a_{1}+\cdots+a_{k}=a_{1}^{\prime}+\cdots+a_{l}^{\prime}+h_{1}+\cdots+h_{l} .
$$

But

$$
a_{1}^{\prime}+\cdots+a_{l}^{\prime}=a_{1}+\cdots+a_{k}-h_{1}-\cdots-h_{l} \in k A,
$$

and this contradicts the fact that $A$ is $(k, l)$-sum-free.

(iii) Since $A \subseteq A+K$ and $A$ is a maximal $(k, l)$-sum-free set in $G$, by (ii) we have $A+K=A$.

(iv) We need to show that for any $a \in A$, we have $a+K \subseteq A$. But $a+K \subseteq A+K$, so the claim follows from (iii).

For the upper bound in Theorem 3, we need the following result which is essentially due to Kneser.

Theorem 8 (Kneser [20]; see Theorem 4.4 in [25]) Suppose that $A$ is a non-empty subset of $G$ and, for a given positive integer $h$, let $H$ be the stabilizer of $h A$. Then we have

$$
|h A| \geq h \cdot|A|-(h-1) \cdot|H| .
$$

Proof of the upper bound in Theorem [3. Let $A$ be a $(k, l)$-sum-free set in $G$ with $|A|=\lambda$; then we have

$$
k A \cap l A=\emptyset
$$

and therefore

$$
n \geq|k A|+|l A| .
$$

As before, let $K$ and $L$ be the stabilizer subgroups of $k A$ and $l A$, respectively. Then, by Theorem 8. we have

$$
|k A| \geq k \cdot|A|-(k-1) \cdot|K|
$$

and

$$
|l A| \geq l \cdot|A|-(l-1) \cdot|L|
$$

thus, from (4) we get

$$
n \geq(k+l) \cdot|A|-(k-1) \cdot|K|-(l-1) \cdot|L| .
$$

Without loss of generality we can assume that $|K| \geq|L|$, so

$$
n \geq(k+l) \cdot|A|-(k+l-2) \cdot|K|
$$


or

$$
\frac{|A|}{|K|} \leq \frac{1}{k+l} \cdot\left(\frac{n}{|K|}+(k+l-2)\right)
$$

Now $|A|=\lambda$; in particular, $A$ is maximal, so by Lemma 7 (iv), $\frac{|A|}{|K|}$ must be an integer. Therefore, with $d$ denoting the index of $K$ in $G$, we get

$$
\frac{\lambda}{n / d} \leq\left\lfloor\frac{1}{k+l} \cdot(d+k+l-2)\right\rfloor
$$

from which our claim follows.

Proposition 9 Let $d$ be a positive integer, and set $\delta(d)=\operatorname{gcd}(d, k-l)$. Suppose that c is a positive integer for which

$$
(k+l) \cdot c \leq d-1-\delta(d) .
$$

Then there exists an element $a \in \mathbb{Z}_{d}$ for which the set

$$
A=\{a, a+1, a+2, \ldots, a+c\}
$$

is a $(k, l)$-sum-free in $\mathbb{Z}_{d}$ of size $c+1$.

Proof. By the Euclidean Algorithm, we have unique integers $q$ and $r$ for which

$$
l \cdot c=\delta(d) \cdot q-r
$$

and $1 \leq r \leq \delta(d)$. We also know the existence of integers $u$ and $v$ for which

$$
\delta(d)=(k-l) \cdot u+d \cdot v .
$$

Now set $a=u \cdot q$. We will show that

$$
A=\{a, a+1, a+2, \ldots, a+c\}
$$

is a $(k, l)$-sum-free in $\mathbb{Z}_{d}$. (Here, and elsewhere, we consider integers as elements of $\mathbb{Z}_{d}$ via the canonical homomorphism $\mathbb{Z} \rightarrow \mathbb{Z}_{d}$.)

First note that, for any integer $i$ with $-l \cdot c \leq i \leq k \cdot c$, our assumption about $c$ implies

$$
1 \leq r \leq l \cdot c+i+r \leq(k+l) \cdot c+r \leq(k+l) \cdot c+\delta(d) \leq d-1,
$$

and therefore, considering

$$
B=\{l \cdot c+i+r \mid-l \cdot c \leq i \leq k \cdot c\}
$$

as a subset of $\mathbb{Z}_{d}$, we have $0 \notin B$.

Furthermore, in $\mathbb{Z}_{d}$ we have

$$
(k-l) \cdot a=(k-l) \cdot u \cdot q=\delta(d) \cdot q-d \cdot v \cdot q=\delta(d) \cdot q=l \cdot c+r,
$$

and therefore

$$
k A-l A=\{(k-l) \cdot a+i \mid-l \cdot c \leq i \leq k \cdot c\}=B .
$$

Since $0 \notin B, A$ is indeed $(k, l)$-sum-free in $\mathbb{Z}_{d}$.

Furthermore, since $c<d$, we see that $|A|=c+1$, as claimed.

The lower bound in Theorem 3 now follows from Proposition 9 and the following lemma. 
Lemma 10 Suppose that $d$ is a divisor of $v$. Then

$$
\lambda_{k, l}(G) \geq \lambda_{k, l}\left(\mathbb{Z}_{d}\right) \cdot \frac{n}{d} .
$$

Proof. Since $d$ is a divisor of $v$, there is a subgroup $H$ of $G$ of index $d$ for which

$$
G / H \cong \mathbb{Z}_{d}
$$

Let $\Phi: G \rightarrow G / H$ be the canonical homomorphism from $G$ to $G / H$, and let $\Psi: G / H \rightarrow \mathbb{Z}_{d}$ be the isomorphism from $G / H$ to $\mathbb{Z}_{d}$. Then, for any $(k, l)$-sum-free set $A \subseteq \mathbb{Z}_{d}$, the set $\Phi^{-1}\left(\Psi^{-1}(A)\right)$ is a $(k, l)$-sum-free set in $G$ and has size $\frac{n}{d} \cdot|A|$.

\section{$3(k, l)$-sum-free sets in cyclic groups}

In this section we analyze $(k, l)$-sum-free arithmetic progressions in $\mathbb{Z}_{n}$ and prove Theorems 4 and 5. This was carried out by Hamidoune and Plagne in [17. with the assumption that $n$ and $k-l$ are relatively prime; here we drop that assumption but follow their approach.

A subset $A$ of $\mathbb{Z}_{n}$ is an arithmetic progression of difference $d \in \mathbb{Z}_{n}$, if

$$
A=\{a, a+d, \ldots, a+c \cdot d\}
$$

for some $a \in \mathbb{Z}_{n}$ and non-negative integer $c$. We let $A_{k, l}(n)$ be the set of $(k, l)$-sum-free arithmetic progression in $\mathbb{Z}_{n}$. We also let $B_{k, l}(n)$ and $C_{k, l}(n)$ be the sets of those sequences in $A_{k, l}(n)$ whose difference is not relatively prime to $n$, and relatively prime to $n$, respectively. Note that a sequence can belong to both $B_{k, l}(n)$ and $C_{k, l}(n)$ only if it contains exactly 1 term, and that sequences in $B_{k, l}(n)$ are each contained in a proper coset in $\mathbb{Z}_{n}$, while no sequence in $C_{k, l}(n)$ with more than one term is contained in a proper coset.

We introduce the following notations.

$$
\begin{aligned}
& \alpha_{k, l}\left(\mathbb{Z}_{n}\right)=\max \left\{|A| \mid A \in A_{k, l}(n)\right\} \\
& \beta_{k, l}\left(\mathbb{Z}_{n}\right)=\max \left\{|A| \mid A \in B_{k, l}(n)\right\} \\
& \gamma_{k, l}\left(\mathbb{Z}_{n}\right)=\max \left\{|A| \mid A \in C_{k, l}(n)\right\}
\end{aligned}
$$

Clearly, $\alpha_{k, l}\left(\mathbb{Z}_{n}\right)=\max \left\{\beta_{k, l}\left(\mathbb{Z}_{n}\right), \gamma_{k, l}\left(\mathbb{Z}_{n}\right)\right\}$.

We also let $D(n)$ be the set of all divisors of $n$ which are greater than 1. Furthermore, we separate the elements of $D(n)$ into subsets $D_{1}(n)$ and $D_{2}(n)$ according to whether they do not or do divide $k-l$, respectively. Then the following are clear:

- $D_{1}(n)=\emptyset$ if, and only if, $k-l$ is divisible by $n$;

- $D_{2}(n)=\emptyset$ if, and only if, $k-l$ and $n$ are relatively prime; and

- $D_{1}(n) \neq \emptyset$ and $D_{2}(n) \neq \emptyset$ if, and only if, $1<\operatorname{gcd}(n, k-l)<n$. 
The next three propositions summarize our results on $\alpha_{k, l}\left(\mathbb{Z}_{n}\right), \beta_{k, l}\left(\mathbb{Z}_{n}\right)$, and $\gamma_{k, l}\left(\mathbb{Z}_{n}\right)$. We start with $\beta_{k, l}\left(\mathbb{Z}_{n}\right)$.

Proposition 11 The maximum size $\beta_{k, l}\left(\mathbb{Z}_{n}\right)$ of a $(k, l)$-sum-free arithmetic progression in $\mathbb{Z}_{n}$ whose difference is not relatively prime to $n$ satisfies the following.

(i) If $k-l$ is divisible by $n$, then $\beta_{k, l}\left(\mathbb{Z}_{n}\right)=0$.

(ii) If $k-l$ and $n$ are relatively prime, then $\beta_{k, l}\left(\mathbb{Z}_{n}\right)=\frac{n}{p}$ where $p$ is the smallest prime divisor of $n$.

(iii) If $1<\operatorname{gcd}(n, k-l)<n$, then we have

$$
\frac{n}{\rho_{1}} \leq \beta_{k, l}\left(\mathbb{Z}_{n}\right) \leq \max \left\{\frac{n}{\rho_{1}}, \frac{n}{2 \rho_{2}}\right\},
$$

where $\rho_{1}$ and $\rho_{2}$ are the smallest elements of $D_{1}(n)$ and $D_{2}(n)$, respectively.

Proof. If $n$ divides $k-l$, then for any $a \in \mathbb{Z}_{n}$ we have $k a=l a$. This implies (i). Statements (ii) and (iii) will follow from the following three claims.

Claim 1. Suppose that $d \in D_{1}(n)$. Then the set

$$
A=\left\{1+i \cdot d \mid 0 \leq i \leq \frac{n}{d}-1\right\}
$$

is an arithmetic progression in $B_{k, l}(n)$, has size $|A|=\frac{n}{d}$, and is $(k, l)$-sum-free.

Proof of Claim 1. Clearly, $A$ belongs to $B_{k, l}(n)$ and has size $|A|=\frac{n}{d}$. Furthermore,

$$
k A-l A=\left\{(k-l)+d \cdot j \mid-l \cdot\left(\frac{n}{d}-1\right) \leq j \leq k \cdot\left(\frac{n}{d}-1\right)\right\} .
$$

Since $d \mid n$ but $d \chi(k-l)$, we have $0 \notin k A-l A$ which means that $A$ is $(k, l)$-sum-free.

Claim 2. Suppose that $H$ is a subgroup of $\mathbb{Z}_{n}$ of index $d$, and that $A$ is a $(k, l)$-sum-free subset of $\mathbb{Z}_{n}$ (not necessarily an arithmetic progression) which lies in a single coset of $H$. Then $|A| \leq \frac{n}{d}$.

Proof of Claim 2. Clearly, $A \subseteq a+H$ implies $|A| \leq|H|=\frac{n}{d}$.

Claim 3. Suppose again that $H$ is a subgroup of $\mathbb{Z}_{n}$ of index $d$, and that $A$ is a $(k, l)$-sum-free subset of $\mathbb{Z}_{n}$ which lies in a single coset of $H$. If $d \in D_{2}(n)$, then $|A| \leq \frac{n}{2 d}$.

Proof of Claim 3. Note that $H$ is a cyclic group of order $n / d$ and

$$
H=\left\{0, d, 2 d, \ldots, \frac{n}{d}-1\right\} .
$$

Since $A$ lies in a single coset of $H$, so do $k A$ and $l A$. But $k-l$ is divisible by $d$, so $k a-l a \in H$, and therefore the sets $k A$ and $l A$ lie in the same coset of $H$. Thus we have

$$
|k A \cup l A| \leq|H|=\frac{n}{d} .
$$

But $A$ is $(k, l)$-sum-free, so $k A$ and $l A$ must be disjoint, hence

$$
|k A|+|l A| \leq \frac{n}{d}
$$


Now clearly $(k-1) a+A \subseteq k A$, so $|A| \leq|k A| ;$ similarly, $|A| \leq|l A|$. This implies that

$$
|A|+|A| \leq \frac{n}{d}
$$

Next, we turn to $\gamma_{k, l}\left(\mathbb{Z}_{n}\right)$.

Proposition 12 The maximum size $\gamma_{k, l}\left(\mathbb{Z}_{n}\right)$ of a $(k, l)$-sum-free arithmetic progression in $\mathbb{Z}_{n}$ whose difference is relatively prime to $n$ satisfies

$$
\left\lfloor\frac{n-1-\delta}{k+l}\right\rfloor+1 \leq \gamma_{k, l}\left(\mathbb{Z}_{n}\right) \leq\left\lfloor\frac{n-2}{k+l}\right\rfloor+1
$$

where $\delta=\operatorname{gcd}(n, k-l)$.

Proof. The lower bound follows directly from Proposition 9,

For the upper bound, suppose that $d \in \mathbb{Z}_{n}$ and $\operatorname{gcd}(d, n)=1$, and let $a \in \mathbb{Z}_{n}$. We need to show that, if the set

$$
A=\{a, a+d, \ldots, a+c \cdot d\}
$$

is $(k, l)$-sum-free in $\mathbb{Z}_{n}$, then

$$
(k+l) \cdot c \leq n-2 .
$$

Suppose, indirectly, that

$$
(k+l) \cdot c \geq n-1
$$

then we have

$$
\{(k-l) \cdot a+i \cdot d \mid-l \cdot c \leq i \leq k \cdot c\} \supseteq\{(k-l) \cdot a+j \cdot d \mid 0 \leq j \leq n-1\} .
$$

Now the left-hand side equals $k A-l A$. Since $\operatorname{gcd}(d, n)=1$, the right-hand side equals the entire group $\mathbb{Z}_{n}$. But then $k A-l A$ must contain 0 , which is a contradiction.

We can now combine Propositions 11 and 12 to get results for the maximum size of $(k, l)$-sum-free arithmetic progressions in $\mathbb{Z}_{n}$.

Proposition 13 The maximum size $\alpha_{k, l}\left(\mathbb{Z}_{n}\right)$ of a $(k, l)$-sum-free arithmetic progression in $\mathbb{Z}_{n}$ satisfies the following.

(i) If $k-l$ is divisible by $n$, then $\alpha_{k, l}\left(\mathbb{Z}_{n}\right)=0$.

(ii) If $k-l$ and $n$ are relatively prime, then

$$
\alpha_{k, l}\left(\mathbb{Z}_{n}\right)=\max \left\{\frac{n}{p},\left\lfloor\frac{n-2}{k+l}\right\rfloor+1\right\}
$$

where $p$ is the smallest prime divisor of $n$.

(iii) If $1<\operatorname{gcd}(n, k-l)<n$, then we have

$$
\max \left\{\frac{n}{\rho_{1}},\left\lfloor\frac{n-1-\delta}{k+l}\right\rfloor+1\right\} \leq \alpha_{k, l}\left(\mathbb{Z}_{n}\right) \leq \max \left\{\frac{n}{\rho_{1}}, \frac{n}{2 \rho_{2}},\left\lfloor\frac{n-2}{k+l}\right\rfloor+1\right\},
$$

where $\delta=\operatorname{gcd}(n, k-l)$, and $\rho_{1}$ and $\rho_{2}$ are the smallest elements of $D_{1}(n)$ and $D_{2}(n)$, respectively. 
It is easy to see that the bounds in Proposition 13 are tight.

Now we are ready to prove Theorem 4, Due to the following result in [17, our task is not difficult.

Theorem 14 (Hamidoune and Plagne, [17]) Let $\epsilon$ be 0 if $n$ is even and 1 if $n$ is odd. Then we have the following bounds.

$$
\max _{d \mid v}\left\{\alpha_{k, l}\left(\mathbb{Z}_{d}\right) \cdot \frac{n}{d}\right\} \leq \lambda_{k, l}(G) \leq \max \left\{\frac{n-\epsilon}{k+l}, \max _{d \mid v}\left\{\alpha_{k, l}\left(\mathbb{Z}_{d}\right) \cdot \frac{n}{d}\right\}\right\}
$$

Proof of Theorem 4. If $k-l$ is divisible by $n$, Theorem 4 obviously holds as both sides equal zero, so let's assume otherwise. By Theorem 14 it suffices to prove that

$$
\left\lfloor\frac{n-\epsilon}{k+l}\right\rfloor \leq \max _{d \mid n}\left\{\alpha_{k, l}\left(\mathbb{Z}_{d}\right) \cdot \frac{n}{d}\right\} .
$$

By Proposition 13, this statement follows once we prove

$$
\left\lfloor\frac{n-\epsilon}{k+l}\right\rfloor \leq \max _{d \mid n}\left\{\max \left\{\frac{d}{\rho_{1}(d)},\left\lfloor\frac{d-1-\delta(d)}{k+l}\right\rfloor+1\right\} \cdot \frac{n}{d}\right\},
$$

where $\rho_{1}(d)$ is the smallest divisor of $d$ which does not divide $k-l$. (Note that in the case when $\delta=1, \rho_{1}(d)$ is simply the smallest prime dividing $d$, thus we do not need to consider cases (ii) and (iii) of Proposition 13 separately.)

Now $\rho_{1}=\rho_{1}(n)$ does not divide $k-l$, so we must have $\delta\left(\rho_{1}\right)=\operatorname{gcd}\left(\rho_{1}, k-l\right)<\rho_{1}$. Therefore, since $\rho_{1}$ divides $n$, we have

$$
\max _{d \mid n}\left\{\left(\left\lfloor\frac{d-1-\delta(d)}{k+l}\right\rfloor+1\right) \cdot \frac{n}{d}\right\} \geq\left(\left\lfloor\frac{\rho_{1}-1-\delta\left(\rho_{1}\right)}{k+l}\right\rfloor+1\right) \cdot \frac{n}{\rho_{1}} \geq \frac{n}{\rho_{1}}
$$

We then have

$$
\begin{aligned}
\max _{d \mid n}\left\{\operatorname { m a x } \left\{\frac{d}{\rho_{1}(d)}\right.\right. & \left.\left.,\left\lfloor\frac{d-1-\delta(d)}{k+l}\right\rfloor+1\right\} \cdot \frac{n}{d}\right\}= \\
& =\max \left\{\max _{d \mid n}\left\{\frac{n}{\rho_{1}(d)}\right\}, \max _{d \mid n}\left\{\left(\left\lfloor\frac{d-1-\delta(d)}{k+l}\right\rfloor+1\right) \cdot \frac{n}{d}\right\}\right\} \\
& =\max \left\{\frac{n}{\rho_{1}}, \max _{d \mid n}\left\{\left(\left\lfloor\frac{d-1-\delta(d)}{k+l}\right\rfloor+1\right) \cdot \frac{n}{d}\right\}\right\} \\
& =\max _{d \mid n}\left\{\left(\left\lfloor\frac{d-1-\delta(d)}{k+l}\right\rfloor+1\right) \cdot \frac{n}{d}\right\} .
\end{aligned}
$$

Therefore, (5) is equivalent to

$$
\left\lfloor\frac{n-\epsilon}{k+l}\right\rfloor \leq \max _{d \mid n}\left\{\left(\left\lfloor\frac{d-1-\delta(d)}{k+l}\right\rfloor+1\right) \cdot \frac{n}{d}\right\} .
$$


But this inequality clearly holds, since

$$
\begin{aligned}
\max _{d \mid n}\left\{\left(\left\lfloor\frac{d-1-\delta(d)}{k+l}\right\rfloor+1\right) \cdot \frac{n}{d}\right\} & \geq\left\lfloor\frac{n-1-\delta}{k+l}\right\rfloor+1 \\
& \geq\left\lfloor\frac{n-1-(k-l)}{k+l}\right\rfloor+1 \\
& =\left\lfloor\frac{n+(2 l-1)}{k+l}\right\rfloor \\
& \geq\left\lfloor\frac{n-\epsilon}{k+l}\right\rfloor .
\end{aligned}
$$

Proof of Theorem 5 . By Theorems 4 and 14 here we need to show that our assumptions imply

$$
\left\lfloor\frac{n-\epsilon}{k+l}\right\rfloor \leq \max _{d \mid v}\left\{\max \left\{\frac{d}{\rho_{1}(d)},\left\lfloor\frac{d-1-\delta(d)}{k+l}\right\rfloor+1\right\} \cdot \frac{n}{d}\right\},
$$

where $\rho_{1}(d)$ is the smallest divisor of $d$ which does not divide $k-l$. (The only difference between (5) and (6) is that in (6) only divisors of $v$ are considered.)

In a similar manner as before, we use the fact that $\rho_{1}(v)$ does not divide $k-l$ to conclude that the right hand side equals

$$
\max _{d \mid v}\left\{\left(\left\lfloor\frac{d-1-\delta(d)}{k+l}\right\rfloor+1\right) \cdot \frac{n}{d}\right\}
$$

Now let $d_{0}$ be a divisor of $v$ which is not congruent to any integer between 1 and $\delta\left(d_{0}\right)$ (inclusive) $(\bmod k+l)$. Then the remainder of $d_{0}-1-\delta\left(d_{0}\right)$ when divided by $k+l$ is at most $k+l-1-\delta\left(d_{0}\right)$. Therefore, we have

$$
\begin{aligned}
\max _{d \mid v}\left\{\left(\left\lfloor\frac{d-1-\delta(d)}{k+l}\right\rfloor+1\right) \cdot \frac{n}{d}\right\} & \geq\left(\left\lfloor\frac{d_{0}-1-\delta\left(d_{0}\right)}{k+l}\right\rfloor+1\right) \cdot \frac{n}{d_{0}} \\
& \geq\left(\frac{d_{0}-(k+l)}{k+l}+1\right) \cdot \frac{n}{d_{0}} \\
& =\frac{n}{k+l},
\end{aligned}
$$

proving ([6).

\section{4 (3, 1)-sum-free sets in cyclic groups}

In this section we prove Theorem 6 and find $\lambda_{3,1}\left(\mathbb{Z}_{n}\right)$ explicitly. First, we evaluate $\alpha_{3,1}\left(\mathbb{Z}_{n}\right)$. We note that, while Proposition 13 (ii) readily yields

$$
\alpha_{2,1}\left(\mathbb{Z}_{n}\right)=\left\{\begin{array}{cll}
\frac{n}{2} & \text { if } & 2 \mid n ; \\
\left\lfloor\frac{n+1}{3}\right\rfloor & \text { if } & 2 \not \chi n
\end{array}\right.
$$

evaluating $\alpha_{3,1}\left(\mathbb{Z}_{n}\right)$ requires a bit more work. 
Proposition 15 The maximum size $\alpha_{3,1}\left(\mathbb{Z}_{n}\right)$ of a $(3,1)$-sum-free arithmetic progression in $\mathbb{Z}_{n}$ is given as follows:

$$
\alpha_{3,1}\left(\mathbb{Z}_{n}\right)=\left\{\begin{array}{cl}
\frac{n}{3} & \text { if } 3 \mid n ; \\
\left\lfloor\frac{n+2}{4}\right\rfloor & \text { if } 3 \not \mid n \text { and } n \not \equiv 2(\bmod 8) ; \\
\frac{n-2}{4} & \text { if } 3 \not \mid n \text { and } n \equiv 2(\bmod 8) .
\end{array}\right.
$$

Proof. Let $\alpha_{3,1}(n)=\alpha$. If $n=2$, the claim holds, so we assume that $n \geq 3$. We distinguish several cases.

Case 1: $2 \not \bigcap n$ and $3 \not \supset n$. In this case Proposition13 (ii) applies, and

$$
\alpha=\left\lfloor\frac{n+2}{4}\right\rfloor .
$$

Case 2: $2 \not\lfloor n$ and $3 \mid n$. Proposition 13 (ii) applies again; we get

$$
\alpha=\max \left\{\frac{n}{3},\left\lfloor\frac{n+2}{4}\right\rfloor\right\}=\frac{n}{3} .
$$

Case 3: $2 \mid n$ and $3 \mid n$. In this case Proposition 13 (iii) applies with $\delta=2, \rho_{1}=3$, and $\rho_{2}=2$; we get

$$
\max \left\{\frac{n}{3},\left\lfloor\frac{n+1}{4}\right\rfloor\right\} \leq \alpha \leq \max \left\{\frac{n}{3}, \frac{n}{4},\left\lfloor\frac{n+2}{4}\right\rfloor\right\},
$$

which again implies

$$
\alpha=\frac{n}{3} .
$$

Case 4: $4 \mid n$ and $3 \not n$. Again Proposition 13 (iii) applies - this time with $\delta=2, \rho_{1}=4$, and $\rho_{2}=2$. Therefore we get

$$
\max \left\{\frac{n}{4},\left\lfloor\frac{n+1}{4}\right\rfloor\right\} \leq \alpha \leq \max \left\{\frac{n}{4},\left\lfloor\frac{n+2}{4}\right\rfloor\right\},
$$

which gives

$$
\alpha=\frac{n}{4} .
$$

Case 5: $n \equiv 2(\bmod 4)$ and $3 \not n$. Again Proposition 13 (iii) applies - this time with $\delta=2$, $\rho_{1} \geq 5$, and $\rho_{2}=2$. Therefore we get

$$
\max \left\{\frac{n}{\rho_{1}},\left\lfloor\frac{n+1}{4}\right\rfloor\right\} \leq \alpha \leq \max \left\{\frac{n}{\rho_{1}}, \frac{n}{4},\left\lfloor\frac{n+2}{4}\right\rfloor\right\},
$$

which yields only

$$
\alpha \in\left\{\frac{n-2}{4}, \frac{n+2}{4}\right\} .
$$

To continue further, we separate the cases of $n \equiv 2(\bmod 8)$ and $n \equiv 6(\bmod 8)$. 
Case 5.1. Let us first consider the case when $n \equiv 6(\bmod 8)$. With $a=\frac{n+2}{8}$ and $c=\frac{n-2}{4}$, we let

$$
A=\{a, a+1, \ldots, a+c\} .
$$

Then

$$
3 A-A=\{2 a-c+i \mid 0 \leq i \leq 4 c\}=\{1+i \mid 0 \leq i \leq n-2\}=\mathbb{Z}_{n} \backslash\{0\},
$$

so $A$ is $(3,1)$-sum-free in $\mathbb{Z}_{n}$ of size $c+1=\frac{n+2}{4}$.

Case 5.2. Now suppose that $n \equiv 2(\bmod 8)$. We prove that $\alpha=\frac{n-2}{4}$. Suppose, indirectly, that $\alpha=\frac{n+2}{4}$ and there is a $(3,1)$-sum-free arithmetic progression

$$
A=\{a, a+d, \ldots, a+c \cdot d\}
$$

in $\mathbb{Z}_{n}$ of size $c+1=\frac{n+2}{4}$. Similarly to above,

$$
3 A-A=\{2 a-c \cdot d+i \cdot d \mid 0 \leq i \leq 4 c\}=\{2 a-c \cdot d+i \cdot d \mid 0 \leq i \leq n-2\} .
$$

By Proposition 11 (iii), we have

$$
\beta_{3,1}(n) \leq \max \left\{\frac{n}{\rho_{1}}, \frac{n}{4}\right\}=\frac{n}{4} ;
$$

so we have $\beta_{3,1}(n)<\alpha$. Therefore, we must have $\operatorname{gcd}(d, n)=1$, which implies that

$$
|3 A-A|=n-1 .
$$

Since $A$ is $(3,1)$-sum-free, $0 \notin 3 A-A$, and this can only occur if

$$
2 a-c \cdot d+(n-1) \cdot d \equiv 0(\bmod n) .
$$

A simple parity argument provides a contradiction: $2 a-c \cdot d+(n-1) \cdot d$ is odd, so it cannot be divisible by $n$.

Proof of Theorem [6. As previously, we let $D(n)$ be the set of divisors of $n$ which are greater than 1. We introduce the following six (potentially empty) subsets of $D(n)$, as well as some notations.

$$
\begin{array}{lll}
E_{1}(n)=\{d \in D(n)|3| d\} & e_{1}=\max _{d \in E_{1}(n)}\left\{\frac{d}{3} \cdot \frac{n}{d}\right\} \\
E_{2}(n)=\{d \in D(n) \mid d \equiv 3(4), 3 \chi \chi d\} & e_{2}=\max _{d \in E_{2}(n)}\left\{\frac{d+1}{4} \cdot \frac{n}{d}\right\} \\
E_{3}(n)=\{d \in D(n)|4| d, 3 \chi d\} & e_{3}=\max _{d \in E_{3}(n)}\left\{\frac{d}{4} \cdot \frac{n}{d}\right\} \\
E_{4}(n)=\{d \in D(n) \mid d \equiv 1(4), 3 \chi d\} & e_{4}=\max _{d \in E_{4}(n)}\left\{\frac{d-1}{4} \cdot \frac{n}{d}\right\} \\
E_{5}(n)=\{d \in D(n) \mid d \equiv 6(8), 3 \chi \chi d\} & e_{5}=\max _{d \in E_{5}(n)}\left\{\frac{d+2}{4} \cdot \frac{n}{d}\right\} \\
E_{6}(n)=\{d \in D(n) \mid d \equiv 2(8), 3 \chi \chi d\} & e_{6}=\max _{d \in E_{6}(n)}\left\{\frac{d-2}{4} \cdot \frac{n}{d}\right\}
\end{array}
$$

(We have the understanding that $\max \emptyset=0$.)

Then we have

$$
D(n)=\cup_{i=1}^{6} E_{i}(n) ;
$$


furthermore, by Theorem 4 and Proposition 15, we have

$$
\lambda_{3,1}\left(\mathbb{Z}_{n}\right)=\max \left\{e_{i} \mid 1 \leq i \leq 6\right\} .
$$

For any $i \in\{1,2, \ldots, 6\}$ for which $E_{i}(n) \neq \emptyset$, we let

$$
p_{i}=\min \left\{E_{i}(n)\right\}
$$

and

$$
n_{i}=\max \left\{E_{i}(n)\right\}
$$

Now suppose that $E_{5}(n) \neq \emptyset$. Then $E_{2}(n) \neq \emptyset$, and $p_{5}=2 \cdot p_{2}$. Therefore

$$
e_{5}=\frac{p_{5}+2}{4} \cdot \frac{n}{p_{5}}=\frac{p_{2}+1}{4} \cdot \frac{n}{p_{2}}=e_{2} .
$$

We can similarly show that, if $E_{6}(n) \neq \emptyset$, then $E_{4}(n) \neq \emptyset$ and $e_{6}=e_{4}$. Therefore, we see that

$$
\lambda_{3,1}\left(\mathbb{Z}_{n}\right)=\max \left\{e_{i} \mid 1 \leq i \leq 4\right\} .
$$

Next, observe that, if $E_{i}(n) \neq \emptyset$ for some $i \in\{1,2,3\}$, then $e_{i} \geq e_{j}$ for all $i<j \leq 4$.

Now we consider the following cases.

Case 1. Suppose that $n$ has divisors which are congruent to $3 \bmod 4$, and let $p$ be the smallest such divisor. If $p=3$, then $E_{1}(n) \neq \emptyset$, thus

$$
\lambda_{3,1}\left(\mathbb{Z}_{n}\right)=e_{1}=\frac{n}{3} .
$$

If, on the other hand, $p>3$, then $E_{1}(n)=\emptyset$ but $E_{2}(n) \neq \emptyset$, thus

$$
\lambda_{3,1}\left(\mathbb{Z}_{n}\right)=e_{2}=\frac{p+1}{p} \cdot \frac{n}{4} .
$$

Case 2. Suppose that $n$ has no divisors which are congruent to $3 \bmod 4$, but that $n$ is divisible by 4 . In this case, $E_{1}(n)=E_{2}(n)=\emptyset$ but $E_{3}(n) \neq \emptyset$, thus

$$
\lambda_{3,1}\left(\mathbb{Z}_{n}\right)=e_{3}=\frac{n}{4} .
$$

Case 3. Suppose that $n$ has no divisors which are congruent to $3 \bmod 4$, and that $n$ is not divisible by 4 . In this case, $E_{1}(n)=E_{2}(n)=E_{3}(n)=\emptyset$ but $E_{4}(n) \neq \emptyset$, thus

$$
\lambda_{3,1}\left(\mathbb{Z}_{n}\right)=e_{4}=\frac{n_{4}-1}{4} \cdot \frac{n}{n_{4}} .
$$

If $n$ is odd, then $n_{4}=n$; if $n$ is even, then (since $n$ is not divisible by 4 ), $n_{4}=\frac{n}{2}$. In either case, we get

$$
\lambda_{3,1}\left(\mathbb{Z}_{n}\right)=e_{4}=\frac{n_{4}-1}{4} \cdot \frac{n}{n_{4}}=\left\lfloor\frac{n}{4}\right\rfloor .
$$

The claims of Theorem 6 now readily follow. 


\section{$5 \quad$ Further comments and open questions}

In this final section, we discuss some interesting open questions.

Our first question is about a possible generalization of Theorems 1 and 6. Note that, according to Theorem 3, we have

$$
\lambda_{k, l}\left(\mathbb{Z}_{n}\right) \leq \max _{d \mid n}\left\{\left(\left\lfloor\frac{d-2}{k+l}\right\rfloor+1\right) \cdot \frac{n}{d}\right\} .
$$

Question 1 Let $D(n)$ be the set of divisors of $n$ (which are greater than 1). Given distinct positive integers $k$ and $l$, is there a subset $D_{k, l}(n)$ of $D(n)$ so that

$$
\lambda_{k, l}\left(\mathbb{Z}_{n}\right)=\max _{d \in D_{k, l}(n)}\left\{\left(\left\lfloor\frac{d-2}{k+l}\right\rfloor+1\right) \cdot \frac{n}{d}\right\} ?
$$

As we see from (2), Question 1 holds with $D_{k, l}(n)=D(n)$ when $n$ and $k-l$ are relatively prime, in particular, for sum-free sets. According to Theorem [6 the set

$$
D_{3,1}(n)=\{d \in D(n) \mid d \not \equiv 2(\bmod 4)\}
$$

works for $(k, l)=(3,1)$. (Note that, if it exists, $D_{k, l}(n)$ is not necessarily unique.)

Moving on to general abelian groups, we observe that, by Lemma 10, we have

$$
\lambda_{k, l}(G) \geq \lambda_{k, l}\left(\mathbb{Z}_{v}\right) \cdot \frac{n}{v} .
$$

Then one of course wonders the following.

Question 2 Given distinct positive integers $k$ and $l$, is

$$
\lambda_{k, l}(G)=\lambda_{k, l}\left(\mathbb{Z}_{v}\right) \cdot \frac{n}{v} ?
$$

According to Theorem 4, Question 2 is equivalent to asking: is

$$
\lambda_{k, l}(G)=\max _{d \mid v}\left\{\alpha_{k, l}\left(\mathbb{Z}_{d}\right) \cdot \frac{n}{d}\right\} ?
$$

Note that Theorem 2 of Green and Ruzsa affirms Question 2 for sum-free sets. Theorem 5 exhibits some other cases when the equality also holds. In particular, as a consequence of Theorem 5. we see that

$$
\lambda_{3,1}(G)=\lambda_{3,1}\left(\mathbb{Z}_{v}\right) \cdot \frac{n}{v}
$$

holds when $v$ (iff $n$ ) has at least one prime divisor which is congruent to $3 \bmod 4$, or when $v$ is divisible by 4 . So the only cases left open are when $v=P$ or $v=2 P$ where $P$ is the product of primes all of whom are congruent to $1 \bmod 4$.

Next, we are interested in characterizing all $(k, l)$-sum-free subsets of maximum size. 
Question 3 What are the $(k, l)$-sum-free subsets $A$ of $G$ with size $|A|=\lambda_{k, l}(G)$ ?

A pleasing answer is given by Bier and Chin [5] for the case when $k \geq 3$ and $G \cong \mathbb{Z}_{p}$ where $p$ is an odd prime: in this case $A$ is an arithmetic progression. The same answer was given by Diananda and Yap [13 earlier for the case when $(k, l)=(2,1)$ (that is, when $A$ is sum-free) and $G \cong \mathbb{Z}_{p}$ with $p$ not congruent to $1 \bmod 3$; however, for $p=3 m+1$ the set

$$
A=\{m, m+2, m+3, \ldots, 2 m-1,2 m+1\}
$$

is also sum-free with maximum size. More generally, the answer to Question 3 is known for $(k, l)=$ $(2,1)$ and when $n$ has at least one divisor not congruent to $1 \bmod 3$ : in this case $A$ is the union of arithmetic progressions of the same length. More precisely, there is a subgroup $H$ in $G$ so that $G / H$ is cyclic and

$$
A=\{(a+H) \cup(a+d+H) \cup \cdots \cup(a+c \cdot d+H)\}
$$

for some $a, d \in G$ and integer $c$. These and other results can be found in 31.

More ambitiously, one may ask for a characterization of all "large" (but not necessarily maximal) $(k, l)$-sum-free sets in $G$. Can one, for example, describe explicitly all $(k, l)$-sum-free sets of size greater than $n /(k+l)$ ? Hamidoune and Plagne [17] carry this out for sum-free sets of size at least $n / 3$ in arbitrary groups. Other results can be found in the papers of Davydov and Tombak [12] and Lev [21], 22].

Our final question is about the number of $(k, l)$-sum-free subsets in $G$, which we here denote by $N_{k, l}(G)$.

Question 4 What is the cardinality $N_{k, l}(G)$ of the set of $(k, l)$-sum-free subsets in $G$ ?

Clearly, any subset of a $(k, l)$-sum-free set is also $(k, l)$-sum-free, so the answer to Question 4 is at least

$$
N_{k, l}(G) \geq 2^{\lambda_{k, l}(G)} .
$$

But there are indications that the number is not much larger. In fact, for sum-free sets we have the following result of Green and Ruzsa [15]:

$$
N_{2,1}(G)=2^{\lambda_{2,1}(G)+o(1) n},
$$

where $o(1)$ approaches zero as $n$ goes to infinity. They have a more accurate approximation for the case when $n$ has a prime divisor which is congruent to $2 \bmod 3$. (This result had been established for even $n$ earlier by Lev, Łuczak, and Schoen [23] and independently by Sapozhenko [28.)

In closing, we mention that the analogues of our questions about the maximum size, the structure, and the number of $(k, l)$-sum-free sets (especially sum-free sets) have been investigated in non-abelian groups (see Kedlaya's papers [18] and [19]) and, more extensively, among the positive integers (see the works of Alon [1], Bilu [6], Calkin [7, Calkin and Taylor [8], Cameron [9], Cameron and Erdös [10] and [11, and Euczak and Schoen [24]). General background references on related questions include Nathanson's book [25], Guy's book [16, and Ruzsa's papers [26] and [27; see also [3] and [4]. 


\section{References}

[1] N. Alon. Independent sets in regular graphs and sum-free subsets of finite groups. Israel J. Math., 73(2) (1991) 247-256.

[2] N. Alon and D. J. Kleitman. Sum-free subsets. In A. Baker, B. Bollobás, and A. Hajnal, editors, A Tribute to Paul Erdős, pages 13-26. Cambridge University Press, Cambridge, 1990.

[3] B. Bajnok. The spanning number and the independence number of a subset of an abelian group. In M. Nathanson, editor, Number theory (New York, 2003), pages 1-16, Springer, New York, 2004.

[4] B. Bajnok and I. Ruzsa. The independence number of a subset of an abelian group. Integers, 3(Paper A2) 2003, 23 pp. (electronic).

[5] T. Bier and A. Y. M. Chin. On $(k, l)$-sets in cyclic groups of odd prime order. Bull. Austral. Math. Soc., 63(1) (2001) 115-121.

[6] Y. Bilu. Sum-free sets and related sets. Combinatorica, 18(4) (1998) 449-459.

[7] N. J. Calkin. On the number of sum-free sets. Bull. London Math. Soc., 22(2) (1990) 141-144.

[8] N. J. Calkin and A. C. Taylor. Counting sets of integers, no $k$ of which sum to another. $J$. Number Theory, 57(2) (1996) 323-327.

[9] P. J. Cameron. Portrait of a typical sum-free set. London Math. Soc. Lecture Note Ser., 123 (1987) 13-42.

[10] P. J. Cameron and P. Erdös. On the number of sets of integers with various properties. Number theory (Banff, AB, 1988), 61-79, 1990.

[11] P. J. Cameron and P. Erdős. Notes on sum-free and related sets. Recent trends in combinatorics (Mátraháza, 1995). Combin. Probab. Comp., 8(1) (1999) 295-107.

[12] A. Davydov and L. Tombak. Quasi-perfect linear binary codes with distance 4 and complete caps in projective geometry. Problemy Peredachi Informatzii, 25(4) (1989) 11-23.

[13] P.H. Diananda and H. P. Yap. Maximal sum-free sets of elements of finite groups. Proceedings of the Japan Academy, 45 (1969) 1-5.

[14] P. Erdős. Extremal problems in number theory. Proc. Sympos. Pure Math., 8:181-189, Amer. Math. Soc., Providence, R.I., 1965.

[15] B. Green and I. Ruzsa. Sum-free sets in abelian groups. Israel J. of Math., 147 (2005) 157-188.

[16] R. K. Guy. Unsolved Problems in Number Theory. Third edition. Springer-Verlag New York, 2004.

[17] Y. o. Hamidoune and A. Plagne. A new critical pair theorem applied to sum-free sets in Abelian groups. Comment. Math. Helv., XX (2003) 1-25.

[18] K. S. Kedlaya. Product-free subsets of groups. Amer. Math. Monthly, 105(10) (1998) 900-906.

[19] K. S. Kedlaya. Large product-free subsets of finite groups. J. Combin. Theory, Ser. A, 77(2) (1997) 339-343. 
[20] M. Kneser. Summenmengen in lokalkompakten abelschen Gruppen. Math. Zeitschr., 66 (1956) $88-110$.

[21] V. F. Lev. Large sum-free sets in ternary spaces. J. Combin. Theory Ser. A, 111(2) (2005) $337-346$.

[22] V. F. Lev. Large sum-free sets in $\mathbb{Z} / p \mathbb{Z}$. Israel J. Math., 154 (2006) 221-233.

[23] V. F. Lev, T. Euczak, and T. Schoen. Sum-free sets in abelian groups. Israel J. Math., 125 (2001) 347-367.

[24] T. Łuczak and T. Schoen. On the maximal density of sum-free sets. Acta Arith., 95(3) (2000) $225-229$.

[25] M. B. Nathanson. Additive Number Theory: Inverse Problems and the Geometry of Sumsets. Springer-Verlag New York, 1996.

[26] I. Ruzsa. Solving linear equations in a set of integers I. Acta Arith., 65(3) (1993) 259-282.

[27] I. Ruzsa. Solving linear equations in a set of integers II. Acta Arith., 72(4) (1995) 385-397.

[28] A. A. Sapozhenko. Asymptotics of the number of sum-free sets in abelian groups of even order. Doklady Akademii Nauk SSSR, 383(4) (2002) 454-457.

[29] T. Schoen. A note on the number of $(k, l)$-sum-free sets. Electron. J. Combin., 7(1) Research Paper 30, (2000) 8pp (electronic).

[30] A. G. Vosper. The critical pairs of subsets of a group of prime order. J. London Math. Soc., 31 (1956) 200-205.

[31] W. D. Wallis, A. P. Street, and J. S. Wallis. Combinatorics: Room Squares, Sum-free Sets, Hadamard Matrices, Lecture Notes in Mathematics, Vol. 292, Part 3. Springer-Verlag, BerlinNew York, 1972. 